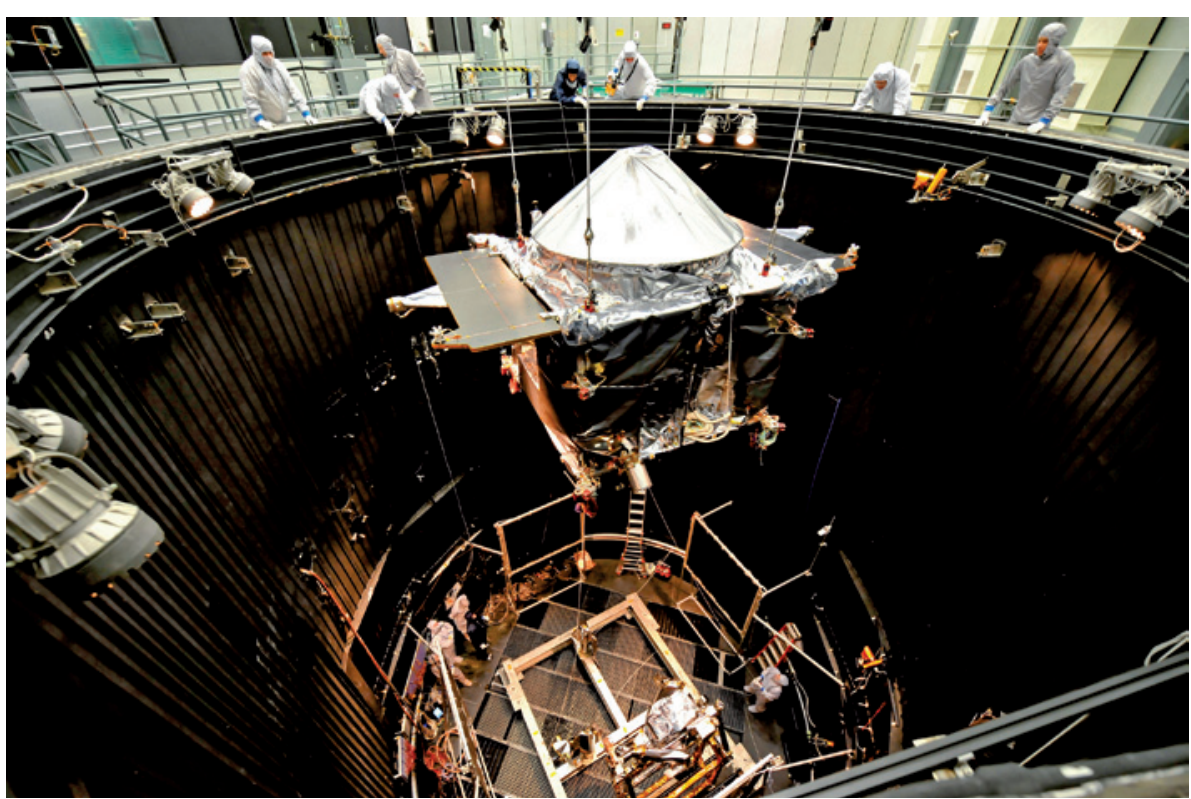

NASA's MAVEN spacecraft will measure the effect of solar storms on Mars' thin outer atmosphere.

PLANETARY SCIENCE

\title{
Mars mission set for launch
}

\section{NASA's MAVEN orbiter aims to unravel the mystery of the red planet's missing atmosphere.}

\section{BY ALEXANDRA WITZE}

$\mathrm{J}$ ust before he submitted a proposal for a mission to Mars to NASA, Bruce Jakosky checked to make sure the word 'aeronomy' hadn't crept into the document. Yes, his satellite would be studying the planet's wispy upper atmosphere, but he knew that NASA would be more interested in the links between the vanishing air and the planet's lost surface water. "We pitched it not as an aeronomy mission, but as a mission to understand the geology," says Jakosky, a planetary geologist at the University of Colorado Boulder.

The strategy worked. In 2008, NASA gave the Mars Atmosphere and Volatile Evolution (MAVEN) mission the go-ahead. The US\$485-million spacecraft is now at Florida's Cape Canaveral, waiting for a 28-day launch window that opens on 18 November. If MAVEN successfully reaches Mars ten months later, it will study how gases in the outer atmosphere escape into space - and how that rate of loss has changed over the past few billion years.

MAVEN comes at a crucial time in NASA's Mars programme. The spacecraft is the only US orbiter planned for the foreseeable future, and it carries the long-term burden of relaying data from NASA rovers on the surface to mission controllers on Earth. The two operating US orbiters, Mars Odyssey and the Mars Reconnaissance Orbiter, were launched in 2001 and 2005, respectively. Of the three, MAVEN is the most likely to be working by the time NASA's next rover heads to Mars in 2020. The MAVEN project was spared from last month's US government shutdown because of this crucial relay ability.

Upon arrival, MAVEN will explore how the solar wind interacts with Mars's upper atmosphere, helping to erode it. "MAVEN's job is to obtain some key constraints that will allow us to make much more solid arguments about how Mars got to be what it is," says Janet Luhmann, a space physicist at the University of California, Berkeley, and the mission's deputy principal investigator.

Canyons carved into the landscape and chemical evidence from rocks indicate that Mars once had a thick atmosphere that kept the surface warm enough for water to flow. But most of that atmosphere is gone; today it is only about $1 \%$ as dense as that of Earth. Mars is so small that the gravitational grip it has on its atmosphere is weak. And when its protec tive global magnetic field switched off, around 4 billion years ago, the planet's hold on its outer atmosphere weakened further.

MAVEN's instruments will explore several of the many ways that atoms in the atmosphere can escape. An ultraviolet spectrograph will measure major components of the atmosphere, including the ratio of deuterium (or 'heavy hydrogen') to hydrogen. The Martian atmosphere is enriched in deuterium because, unlike lighter-weight hydrogen, the isotope had insufficient thermal energy to break free of the planet's gravitational pull. Comparing the ratio of deuterium to hydrogen in the atmosphere with the ratio locked in ancient rocks at the planet's surface should reveal the rate of this particular type of loss over time.

Other missions to Mars have studied another type of loss: the depletion of charged particles. In 1988, the Soviet Phobos- 2 mission made the first detailed measurements showing that the solar wind stripped ions from Mars's atmosphere. By 2007, the European Space Agency's Mars Express orbiter had discovered that several grams of oxygen and carbon dioxide ions were being lost each second (S. Barabash et al. Science 315, 501-503; 2007).

Unlike these missions, MAVEN will be able to study a third type of escape: the erosion of neutral particles, such as hot oxygen atoms, that form in the upper atmosphere and escape because of their high energies. In addition, MAVEN will be able to study changes in extreme ultraviolet light flooding from the Sun, and watch for differences in the solar wind caused by solar storms, to measure the effect of solar activity on atmospheric losses. Together, these measurements should paint a more detailed picture of atmospheric escape than ever before, says Jakosky.

Mars Express will also continue to contribute. Its researchers and those working on MAVEN are planning to make simultaneous observations with the two spacecraft. When both are in place, their elliptical paths will take them from a few hundred kilometres above the planet's surface to thousands of kilometres away. While one of the probes is deep in the atmosphere, the other will have a broader perspective from afar. "Having two spacecraft capable of focusing on the same subject is very exciting," says Stas Barabash at the Swedish Institute of Space Physics in Kiruna, a member of the Mars Express science team.

Mars Express has funds to operate until the end of 2016. India's first Mars mission, a methane-sniffing orbiter that launched on 5 November, will also overlap with MAVEN: both spacecraft are due to arrive at Mars in the same month.

MAVEN has a one-year nominal mission in which to accomplish its goals. But as it fulfils its longer-term communications tasks through the years, it may be well placed to watch the Martian atmosphere eroding before its electronic eyes. 